Clara Stella

\title{
Tra «infiammate stille» e «lacci cari»: la Cecilia di Margherita Costa (1644)
}

\begin{abstract}
Il Cecilia Martire è stampato nel 1644 e dedicato al cardinale Francesco Barberini. Costa, in cerca di un nuovo protettore, si cimenta per la prima volta in un poema sacro. Il saggio si sofferma sui momenti più virtuosistici di Costa e sulla linea erotica che caratterizza il percorso, quasi mistico, di Cecilia. Dall'esile passio di Cecilia, Costa crea un poema diviso in quattro canti che segue le fasi del martirio della giovane, selezionando i quadri più drammatici in una grande ecfrasi letteraria che si relaziona anche ai lavori di ristrutturazione della basilica di Santa Cecilia in Trastevere, sorta sulla presunta domus della martire. I quadri vivono del crescere dell'eroicità di Cecilia, intensificata dall'ambiente infernale che si crea intorno alla giovane e che l'autrice modella sapientemente sull'Inferno dantesco, su Tasso e Marino, ma anche su opere coeve di impianto scientifico, come gli scritti sull'eruzione dopo il disastro del Vesuvio nel 1631. Infine, il personaggio della Sofronia tassiana è certamente un punto di riferimento per Costa nella costruzione di Cecilia e del suo rapporto con Valeriano, il cui erotismo sconfina nel profano nelle ottave espunte dalla stampa ma ancora leggibili nel ms. Vat. Barb. Lat. 4069 e di cui il saggio offre una lettura mirata.
\end{abstract}

The e-journal «altrelettere» is hosted at the URL: http://www.altrelettere.uzh.ch, in accordance with the Open Access Policy of the University of Zurich. Please cite this article as follows: C. STELLA, Tra «infiammate stille» $e$ «lacci cari»: la Cecilia di Margherita Costa (1644), in «altrelettere», (2021), pp. 94-116, DOI: 10.5903/al_uzh-55.

(C) This article is licensed under a Creative Commons Attribution 2.5. Switzerland (CC BY-NC-ND 2.5). Please read the license terms on the website: http://creativecommons.org/licenses/by-nc-nd/2.5/ch/deed.en 
C. STELLA, Tra «infiammate stille» e «lacci cari»: la Cecilia di Margherita Costa (1644), in «altrelettere», (2021), 95 pp. 94-116, DOI: 10.5903/al_uzh-55.

\section{Introduzione}

Come scrive bene Jessica Goethals, la figura di santa Cecilia a differenza di altre sante come Giuditta o Ester, intrinsecamente drammatiche poiché legate alla violenza subita e perpetrata, è stata a lungo dimenticata nel Pantheon delle eroine cristiane (GoETHALS 2020a). ${ }^{1} \mathrm{Al}$ contrario, anche la mite Cecilia ha offerto molteplici spunti in ambito letterario e in quello artistico: temi e motivi dal racconto della passio della giovane trasteverina vennero impiegati a piacimento sul palcoscenico, sulla tela e su carta, per interpretare in quadri, drammi teatrali ed operistici il suo soggetto (RUSSANO HANNING 2004). ${ }^{2}$ Seguendo la fortuna agiografica della sua storia, più della metà delle pubblicazioni sulla vita di Cecilia appaiono, non a caso, nella prima metà del Seicento, e questo dato sarà da connettere alla fastosa riscoperta della tomba della martire nella basilica di Santa Cecilia in Trastevere nel $1599:^{3}$ un fatto straordinario che, poco prima del Giubileo del 1600, «spurred textual excitement about her for several decades in both sacred and secular circles» (Goethals 2020a, 50). ${ }^{4}$ Margherita Costa si avvalse della rinnovata fortuna di Cecilia per inscenare, e avallare artisticamente, un pentimento di pubblico dominio, esprimendo il suo talento nel nuovo genere dell'epica agiografica con un poema in quattro canti dedicato al cardinale Francesco Barberini, ovvero il Cecilia martire (CosTA 1644), smaccatamente encomiastico della genealogia della corrente famiglia papale. ${ }^{5}$

Due dei momenti fondamentali della vita di Costa, l'arrivo cioè a Firenze e a Roma, si accompagnarono a nuove rinascite e invenzioni artistiche, ed è ormai chiaro alla critica come l'autrice abbia saputo lavorare, negli anni, con la sua immagine, adattandola alle proprie necessità e ai bisogni del pubblico e della committenza (GoETHALS 2017a). Le due città sono quindi associabili a due modelli di ispirazione lirica che provengono dalla bizzarria, dalla buffoneria, dalla sensualità e dal meraviglioso, mediati, questi ultimi, dal programma classicizzante dei cigni della corte romana diretti dal papa-poeta Urbano VIII. La forma del poema agiografico le permise di esibire un allineamento strategico allo stile della riforma poetica proposta dai Barberini che, idealmente, volevano porsi come alternativa all'Adone di Marino, condannato all'indice da Urbano VIII (CARMINATI 2018, 42), con il dispiegamento di un esercito di nuovi eroi, i martiri della cristianità, e un linguaggio nuovo, sacro e piacevole allo stesso 
tempo, che riscrivesse l'elemento religioso attingendo dalla tradizione lirica profana ma ispirandosi alle punte più mistiche e sensuali del testo biblico (Fumaroli 1994, 149). Nel Cecilia martire Costa riesce a creare un piccolo gioiello, giocando sul piano del linguaggio e della trattazione della materia agiografica su più livelli. Il poema, che deriva la sua suddivisione in quattro canti da La strage degli innocenti di Marino, dilata e accorcia la trama della passio latina in modo sostanziale e si concentra, a differenza delle riscritture precedenti in chiave epica, sui momenti centrali del martirio (Il Bagno e $I l$ Martirio), per poi dare spazio alle figure papali nel terzo canto (Il Tempio) e ritornare, infine, a descrivere la trasformazione della casa di Cecilia da domus a tempio sepolcrale nell'ultimo quadro (Il Sepolcro). ${ }^{6}$

Il Cecilia martire di Costa non è una storia in versi, o una semplice e didascalica ripresa della vicenda agiografica, ma un vero e proprio poema i cui marcatori epici sono attentamente esibiti dall'autrice. Costa non lascia spazio al racconto, ad esempio, del matrimonio con Valeriano, oggetto di fortunati drammi teatrali a partire da quello di Suor Cherubina Venturelli (WEAVER 2009, 37-51), né alla conversione del marito e del cognato, e neppure alla carità di Cecilia, la quale, per tre giorni, dopo essere stata decapitata, avrebbe continuato a convertire i compaesani e distribuito i suoi beni ai poveri. I punti più narrativi della passio sono dismessi per quelli più scenografici ed epici: lo scontro tra le forze paradisiache e quelle infernali, ad esempio, aperto dal concistoro presieduto da Plutone nel canto I, occupa uno spazio centrale e crea lo scenario entro cui Costa incastona l'encomio del papato Barberini. Tralasciando i complessi intenti celebrativi del poema, in questo saggio si metteranno in rilievo, invece, i punti che caratterizzano la linea virtuosistica ed erotica di Costa, esaminando in particolare la rappresentazione del tripudio di fuoco del balneum (cfr. Costa-ZALESSOW 2015, 34, 209), il rapporto con l'elemento ecfrastico e la riscrittura della vicenda tassiana di Sofronia e Olindo in chiave epico-agiografica. In quest'ultimo caso, infatti, nel tratteggiare il rapporto amoroso tra Valeriano e Cecilia, Costa poté ispirarsi alla galleria di amanti rifiutati delineata nelle sue Lettere amorose del 1639, ristampate nel 1643 (CostA 1639). È probabilmente sulla loro voce che modella alcune ottave amorose che verranno successivamente espunte dall'edizione a stampa del poema, ma che ancora si leggono in una bella copia del poema nel ms. Vat. Barb. 
C. STELLA, Tra «infiammate stille» e «lacci cari»: la Cecilia di Margherita Costa (1644), in «altrelettere», (2021), 97 pp. 94-116, DOI: 10.5903/al_uzh-55.

Lat. 4069 (COSTA-ZALESSOW 2015, 35) di uso privato e certamente mai eliminata dagli scaffali della biblioteca del cardinale dedicatario Francesco Barberini.

\section{Lo spazio del divertissement: dal Bagno al Sepolcro}

Nel canto I l'edificio che si apre al lettore è ridotto in «vampe», l'aria «stride» e i bollori e i fumi che il miscuglio di aria, fuoco e ghiaccio creano rendono la domus di Cecilia simile a un paesaggio infernale (I, 13-16). Come in un grande teatro le tende delle pareti del «Palagio» si aprono, in medias res, sull'inferno dei vapori ardenti del martirio («stanza più non somiglia; e fatta è reggia / di terremoti, ov' il furor fiammeggia»; I, 55). Qui entra in scena Cecilia che «la lingua discioglie in dolci note» $(I, 17)$ per esortare le fiamme a crescere ancora più vive e accese, costruendo un parallelismo con il canto intonato nelle ottave proemiali dalla voce di una Costa penitente e che si potrà seguire lungo il poema, riavvolgendo il filo che unisce il tema della musica, del canto e della performance della «diva» Cecilia. ${ }^{7}$

La descrizione dei vapori e dei fumi che inondano il balneum è resa da Costa dettagliatamente, con un progressivo climax che pone l'enfasi sulle percezioni sonore e sensoriali del fenomeno. Il fumo, il vapore e il calore sono descritti come i pronostici di un'eruzione vulcanica che si incanala, con la sua potenza e forza, nello spazio angusto del piccolo bagno della giovane martire. Un frastuono «di catene, di ferri, e d'urli» che fa «risonar»e «rimbombare» il tutto (I, 42) annuncia l'arrivo del fuoco in colate di lava ed emanazioni sulfureomagmatiche prodotte direttamente dalla fucina di Efesto in «Mongibello» (I, 45), cioè nell'Etna. Tasso, nel Mondo creato (III, 752-69, in TASSO [1951]) aveva inserito una descrizione del percorso tortuoso delle acque termali che, procedendo dal mare, «per oscure, e tenebrose strade / sotto la cavernosa e rara terra» si incanalano («quasi per mine occulte assai profonde / e poi che da se stesso in lor s’è chiuso / con gli obliqui suoi corsi ascende in alto»), zampillando poi in superficie sotto forma di acqua calda e solforosa («e de' purgati umori / il terrestre amator cangiat' ha 'n dolce, / e trapassando da' metalli ei prende / qualità viepiù calda, onde sovente / con fervid'acque egli s'accende, e bolle»). Marino aveva ripreso il motivo delle acque termali in chiave erotica in una coppia di sonetti delle Rime amorose che ritraggono la donna amata alla 
«Solfaia» di Pozzuolo (MARINo [1987], 179), ${ }^{8}$ descritta come luogo infernale, e di cui riportiamo la prima parte, altamente evocativa del Tasso del Mondo creato:

Là, dove Stige per sulfurea vena di fiamme inestinguibili e di fumi rapidi globbi e torbidi volumi manda a turbar la pura aria serena, e donde van per infeconda arena con tacito bollor pallidi fiumi, e fra sanguigne nebbie e ciechi lumi mirasi l'uscio de l'eterna pena, cruda donna sen vien, per mirar quivi fra le perdute e desperate genti mill'amanti per lei di vita privi (MARINO [1987], n. 72).

Il lessico del fuoco di Costa è affine alla declinazione solfureo-amorosa di Marino, con la quale converge per segmenti lessicali: il pezzo virtuosistico dell'autrice, giocato sulla variazione, ha comunque una base archetipica nella Commedia dantesca, i cui luoghi infernali erano ritornati in auge con l'eruzione dell'Etna del 1631, la cui portata sentita quasi come "apocalittica" diede argomento cronachistico e poetico a molti autori soprattutto napoletani (RICCIO 1889, 513-21). Il fuoco dell'Etna è invocato da Plutone a riscaldare l'acqua del martirio di Cecilia con eco del XIV dell'Inferno: lì «in Mongibello» era stata posta la «focina negra» (ALIGHIERI [1966-1967], Inf. XIV, 56), cioè quella casa dei Ciclopi che già la letteratura sull'eruzione del Vesuvio aveva fatto propria. Anche la stanzetta di Cecilia, trasformatasi in un convoluto meccanismo ingegneristico dove l'acqua si tramuta in faville ardenti attraverso i tubi e le bocchette da cui poi fuoriesce, diventa, iperbolicamente, il peggiore dei luoghi infernali, l'«isola ardente» di Vulcano dove «tutti i Mostri d'Averno» (I, 49) trovano la loro sede per «l'interno suo foco ogni or lucente»:

Spruzzano gli empi d'infiammate stille le pure membra e di cocenti umori spandon sovra lei calde faville, e dentr' a l'acqua nudrono bollori 
par, che tolti da l'Orco a cento, a mille

v'avventin di Vulcano i rei furori

e di Plutone la region concente

ivi rinovi un Mongibello ardente (I, 45).

Austro infernal con mormorar'orrendo

ale spande di foco e 'l luogo ingombra,

indi quell'acque co 'l suo soffio aprendo,

dal fondo l'alza e una tempesta adombra:

l'umor in sé ricade e ogni or tremendo

il furor più s'avanza in sen' a l'ombra:

ondeggian co' tremori i flutti sparsi,

e tutti gli elementi ivi son' arsi (I, 48).

L'aria è scossa da uno «spaventoso suono» che indica l'imminenza di una tempesta, impastata di «grave [...] nebbia» e di «tempestoso umor» (I, 52), che si scatena sopra Cecilia e l'archetipo non può che essere la pioggia dantesca mista di caligine e fuoco di Inferno XIV (vv. 1-42). Le nubi preannunciano la conflagrazione funesta di tutti gli elementi naturali («di lampi e di saette il cielo tutto / annuntia fra tempeste eterno lutto»; I, 53): solo a questo punto si alzano le onde, che fuoriescono, miste di vapore, dai lati delle pareti con «muggir spietato» $(\mathrm{I}, 54)$. Costa, così facendo, rende in forma poetica quell'emissione di vapore acqueo che il cardinale Paolo Emilio Sfondrati si era immaginato uscire dalle bocchette della stanza di Cecilia, riemerse grazie gli scavi nel luogo del martirio, divenuto per volontà del cardinale la cosiddetta Cappella del Bagno della basilica trasteverina:

S'alza l'acqua cocente e 'l dorso irato inver le stelle sollevar si scorge, or' esce fuori del ristretto lato, ed or, per ruinare, in alto sorge: fervida stride e con muggir spietato fuor de l'usata sponda i flutti porge e spruzza e spande fra l'ardenti stille fiamme, fumo, terror, gelo e faville (I, 54).

Un terribile terremoto conclude la scena: attorno a Cecilia ruotano «a cento, a mille / i folgori» e sia le mura che i marmi del palazzo «versano scintille», avvolgendola tutt'attorno (I, 56). Sul calore del fuoco e la costanza di Cecilia si 
gioca il contrasto tra la morte e la vita eterna: il turbinio dei venti e le onde infuocate sono per la giovane martire un vento di refrigerio, e il motivo poetico sopra il quale Costa può ricamare una serie di contrasti tra il paesaggio infernale e il piacere delle membra candide di Cecilia a contatto con il fuoco, l'aria infuocata e l'acqua bollente. Qui entra in scena una martire suadente, che sprigiona bellezza, sensualità e piacere, oggetto della parte finale di questo contributo.

Se «l'albergo ch'arse orride facelle» (III, 102) era stato, nei canti I e II, un luogo d'inferno, la vittoria di Cecilia e di Urbano I sul male porta a un teatrale cambio di scena: il canto IV, ovvero Il Sepolcro, infatti, è un tripudio di gemme, pietre preziose e addobbi paradisiaci: Urbano I, al quale è affidata la cura del corpo di Cecilia dopo la morte, trasforma la domus romana della giovane in un luogo di culto. Questa è l'occasione, per Costa, di un affondo ecfrastico che risponde effettivamente alla preziosità della Cappella del Bagno e ai marmi e alle pietre che adornano la tomba della martire, posta nel presbiterio, dove è incastonata la fine statua di Maderno, voluta da Sfondrati e realizzata ad inizio secolo (GOODSON 2007; RADEGLIA 2007). Alla luce dei raggi solari la tomba risplende delle più belle gemme del mondo: il diamante, il carbonchio, lo zaffiro, il crisolito, la granata, l'ametista. Personificate, infatti, le «gemme tra lor» fanno «gare d'onore»: avvampano e accrescono la loro bellezza non appena toccate dallo sguardo altrui («per meraviglia di chi 'l guardo gira», IV, 54). Il pavimento è tempestato di smeraldi e la porta della domus viene arricchita, dagli angeli, di alloro, perle e oro: la luce è tale che «un novo giorno ed un novello cielo» $(\mathrm{IV}, 58)$ sembrano prendere vita racchiusi nella basilica. Il ruolo della musica, anche in quest'opera di Costa, è centrale: Dio è accolto in questo paradiso in terra con «tenzoni care» e «festosi gli augelletti in varii accenti / giungon al nobil pregio alti concenti» (IV, 60) ma, soprattutto, il momento è salutato da un'armonia cosmica data dal silenzio dei Cieli, che permette di amplificare, come una cassa di risonanza, il tripudio degli inni angelici. Gli angeli, in processione, scendono in terra e sono rappresentati da Costa come una «corte di stelle» che «in terra move / i piè di perle, e i vanni d'oro» lungo la stanza (IV, 64). L'angelo custode di Cecilia le pone sopra il capo, infine, un velo bianco, trapuntato di diamanti e rubini, e direzionandole il volto dall'altro lato come nella posa della statua di Maderno. Per salutare questo gesto, di 
nuovo, le corti angeliche rinnovano il canto «a cento a mille» in una nuova rinascita primaverile di suoni e profumi (IV, 67). In queste ottave, che anticipano il pomposo corteo funebre che accompagnerà la salma di Cecilia lungo la via Appia verso il primo luogo della sua sepoltura, Costa riscrive in chiave femminile la tradizione epica del corteo e dei giochi funebri: questa volta gli onori non sono tributati a degli eroi del mito o della storia, ma ad una semplice «giovinetta» che, come esclama il cognato Tiburzio, ha avuto il merito di abbassare l'onore e la superbia degli uomini in terra e in cielo («per te converso de' nocenti mali / rintuzzi contra noi l’ingiusto orgoglio» IV, 41).

\section{Cecilia martire e Cecilia tentatrice nelle ottave riscritte}

Proprio il moto di ribellione di Cecilia dà il la alla macchina bellica su modello dell'Eneide, e nell'ultimo canto le parole di Urbano metteranno la giovane di fronte agli esempi di tutte quelle «molte Vergini» che «ancora hanno disgiunto / dal corpo l'alma a la salute intese, / bench' in tenera etade e Dio lor diede / tra i regni de le stelle aver la fede», come Tecla e Felicita che ora stanno come fenici «ferme invariabili» fisse attorno al gran «Motore» e lungo la via Appia (III, 6869). Plutone è offeso, infatti, che «da femina Averno oppresso sia» (I, 87) e dalla sua prospettiva Cecilia pecca di superbia e di ardire per la resistenza che dimostra nella difesa della propria castità, poiché, secondo un criterio profano, «vita non merta chi l'amor non prezza». Ancor più, per Plutone, la castità matrimoniale di Cecilia è un negare «fede a la fede», è un non adempiere al dovere fondamentale di ogni sposa, quello cioè di «consumar gli atti amorosi» $(I, 86)$. Le furie continueranno in questo vilipendio definendo Cecilia, in termini misogini, come una «donzella», una «forsennata fanciulla» e una «fragil verginella» che «sprezza Pluto e vilipende Aletto» con l'assurdità dei suoi voti (I, 86-87).

Nel secondo canto l'ira di Almachio risvegliato dalle Furie lo rende «tra mostri un mostro» (II, 5), al punto da farlo assomigliare a Pluto e indurlo a chiamare un boia per giustiziare, con decollazione, Cecilia. La sua trasformazione da uomo a giudice iracondo e poi a vera fera infernale utilizza la fenomenologia dantesca, già declinata negli interpreti del male della Strage degli innocenti di Marino (Satana, Erode e i carnefici): spaventato dalla sua 
stessa immagine, «agitato si vede, e nudo e inerme / sé stesso incontra, e sé medesmo aborre» (II, 9). Costa descrive la figura di Almachio come una creatura diabolica e irrazionale tramite un gioco di antitesi, iperboli e rime inclusive per raffigurare gli aspetti contrastanti e violenti della sua trasformazione: «confusi gli accenti escon da lui / e di pavido ghiaccio il petto cinge», «tra Furie rassembra e larva e furia / ch'agitata da Pluto agita e infuria» (II, 13). Al nascere del giorno Almachio è privo di vita e intelletto («semivivo e senza spirto» II, 14) in contrasto con l'Almachio-retore e giudice della passio che, seppur antitetico a Cecilia, aveva mostrato capacità d'analisi sottoponendo tutti e tre i martiri, inclusa la giovane, a un interrogatorio. Anche secondo Almachio Cecilia è una «vil femminuccia» e una delle «cristiane ancelle» che «tant'osano» e tanto sono orgogliose della loro fede contraria agli dei. Cecilia, allora, è in odio a Pluto e ad Almachio non solo per la sua fede costante, ma anche per il suo essere una donna che sfida gli ordini precostituiti:

Ed ora una donzella, un sesso vile, ch'ha ne la fresca età molle germoglio, abbatterà con temerario stile del forte imperador l'invitto soglio? Ah 'l suo vanto per me si prenda à vile, e con la vita il feminile orgoglio estinto manchi: e a questo dir nel core e tutt'odio, tutt'ira, e tutto orrore (II, 25).

Se il contrasto Plutone-Almachio-Cecilia può ricordare le posizioni retoriche della questione della donna, l'archetipo epico-letterario di Costa è quello della Sofronia tassiana, guerriera senz'armi che usa la castità come difesa ed elemento di indipendenza ma la cui estraneità al modello epico è sottolineata da Tasso in più punti (BENEDETTI 1997, 97-108). Sofronia non è giovane, ma è descritta di già «matura / verginità», inavvicinabile, chiusa in sé come una città fortificata irraggiungibile (TASSO [2009], II.14). Nel poema tassiano la castità di Sofronia non vive di un'apoteosi, ma è letta, al contrario, in un moto di intransigenza non contemplato nell'economia del poema, e che è punito dal suo autore e dalla logica del bene comune con l'imposizione forzata del matrimonio con Olindo. Sofronia, che non è investita da Dio, non è nel luogo letterario 
adatto per poter vincere sulla storia e, per questo, sarà oggetto di numerosi ripensamenti da parte di Tasso che finirà per escludere l'episodio dei due amanti nella Conquistata (BENEDETTI 1997, 114-15).

Le diverse muse che ispirano la poesia d'amore di Costa sono, di fatto, portatrici di due differenti concezioni dell'erotico: quello sensuale e terreno esemplificato da Simona, la musa de La Chitarra, (CosTA 1638, 3) e quello sublimato e cristiano di Cecilia. Le parole di quest'ultima escono da un «purpureo labro», il suono è «dolce a l'aria» come «aure odorose» (I, 76): le caratteristiche delle tessere petrarchistiche si sposano con gli attributi della santità della vergine romana (cfr. BELLINI 2004, 167-97). La sensualità, che si concentrata nelle varie fasi del martirio di Cecilia, che «di vapori celesti» avvampa e arde (I, 71), non trabocca in licenziosità ma, come ribadisce la voce narrante, è espressione di una «fede» ardente che si accresce nelle «onde»e pulsa di «vene amorose» (I, 76). La figura di Cecilia-martire apre a Costa la possibilità di confrontarsi non solo con i poli privilegiati della poesia alta, l'epica agiografica e la tradizione della virgo militans, ma di continuare a sperimentare con il tema di eros e thanatos e di inscrivere l'opera nel linguaggio dei poeti della corte barberina sacralizzando l'erotico ed erotizzando il sacro.

Nel poemetto l'elemento erotico è principalmente declinato all'interno dell'esperienza corporea di Cecilia. In questo senso, la Cecilia letteraria di Costa gareggia con la statua di Maderno, che aveva tradotto in marmo la morte di Cecilia come un sonno perpetuo e delicato e per questo molto più "vero" della vita stessa. L'artista, che aveva già realizzato un'interpretazione carmelitana dell'estasi di Brigida di Svezia, inginocchiata nella cappella confessionale della basilica di San Paolo fuori le mura, realizza con Cecilia un ideale di purezza e di cristianesimo primitivo su archetipi classici (Lo BIANCO 2007, 163). Circa vent'anni dopo, il linguaggio marmoreo di Bernini avrebbe creato e aperto, invece, nuove visioni con la realizzazione del complesso di Dafne ed Apollo, su committenza privata dei Barberini, e dell'estasi di santa Teresa d'Avila, i cui lavori iniziarono tra il 1644 e il 1645 (cfr. BELLINI 2009, 165-76). Se questi sono i macro-poli dell'arte figurativa, Costa ha davanti a sé la possibilità di descrivere i tormenti e l'infuocato coraggio di Cecilia traendo ispirazione dalla sensualità del misticismo seicentesco e dalla bellezza verginale delle sue protagoniste. 
Riprendendo la figura dell'apostola innamorata Maddalena, e, soprattutto, l'erotismo delle mistiche, Cecilia è più volte ritratta come un' «accesa amante» che «gode» dello spettacolo infernale che le si costruisce attorno (I, 58). ${ }^{9}$ In Costa, la verginità e la fedeltà ai valori cristiani sono esaltate con la descrizione dell'accrescimento della bellezza e del piacere che aumentano esponenzialmente all'acuirsi delle pene. Secondo un topos mariano la bellezza è lo specchio della purezza di Cecilia, che diventa più cristallina a mano a mano che le fiamme l'avvolgono; ma Costa si avvale anche della tradizione petrarchistica, a cui associa il lessico dell'esperienza spirituale. Armata del suo «molle seno», Cecilia ha il viso «sereno», sente il fuoco come un «dolce letto» su cui adagiarsi e la pena è un «dilettevole gioco» e una «dolce prova» (I, 60); inoltre, il piacere e il godimento sensuale dell'unione con Dio sono lo spazio entro cui si innalzano i suoi inni di lode che invocano l'intensificarsi del martirio:

Sì sì stille, sì sì vampe crescete abbruciatemi il seno, ardo d'amore; fiammatemi l'alma, il petto ardete sono lieve le fiamme a tanto core; voi con foco maggior non m'accendete, di quello, onde m'accese il mio Signore (I, 65).

Nel fuoco, allora - forma nella quale si manifestò Dio a Mosè ed elemento del martirio di Elia e dell'incendio «dei fanciulli di Babelle», che Cecilia ricorda nel primo discorso pronunciato in apertura del Canto I $(\mathrm{I}, 19)$ - la parola può snodarsi e trarre la forza di cantare il più «armonioso» dei suoi canti (I, 22). Cecilia, in questo senso, è un'eroina controriformistica che non conosce altro senso se non quello della missione di morire come «sposa ancella» e amante di Cristo, aspettando di essere trafitta solamente dal suo dardo («trafigga il petto mio più fiero dardo, / ed infiammi il mio sen più ardente foco »; II, 72), come la Teresa d'Avila che muore «tutta infiammata di grande amore di Dio» (AviLA [1998], 328-29). Costa scolpisce con le sue parole un modello di eroina, martire circoscritta dalla sua missione, che sfida gli elementi della natura per il proprio piacere, mettendo al centro il suo potere di resistenza. 
Il riconoscimento della correttezza della scelta di castità di Cecilia da parte del marito Valeriano, che prende la parola nell'ultimo canto lodando la moglie, seguito a ruota dal fratello Tiburzio, è un tema legato alle riflessioni controriformistiche sul matrimonio, propugnate dalla stessa stamperia vaticana almeno a partire dal 1565 , anno in cui si erano fatte pubblicare le lettere di san Girolamo sulla verginità e la castità matrimoniale (BARBERI 1942, 111-17). Il corpo puro, intatto e intriso di «vera» vita di Cecilia, giace a terra ed è il fulcro dello sguardo dei due fratelli, che si precipitano dal Cielo in terra a renderle onore, riprendendo in questo viaggio sembianza umana («benché le sue membra in lei sien morte, / ha pur d'ogni virtù forma vitale / e par che dica ogniuno "oh non è morta, / oh da la morte sua quivi è risorta" », IV, 9). L'enfasi sulla ferita che spicca sul candore marmoreo del collo di Cecilia non può che ricordare il colore e la virginale compostezza della statua di Maderno:

Ma Valeriano, ch'a lei visse unito
di giogo marital fra lacci cari,
volge, di moglie pia grato marito,
ver lei d'eternitade i lumi chiari:
e visto da tre colpi esser partito
quel collo, ch'adeguar de' marmi pari
puote in candori l'eccellenza pura,
e rende al suo splendor la neve oscura $(I V, 10)$.

Nella versione a stampa, Valeriano ripercorre il martirio di Cecilia ribadendone il significato all'interno del disegno divino: il martirio del bagno «di macchie i corpi lava, / e d'ogni impurità mondi ne rende» continuando la felice sovrapposizione tra i significati dell'acqua, elemento di morte e di vita insieme, nella vicenda di Cecilia (IV, 15). La fiamma è gradita ai martiri perché purifica l'anima e il corpo, e i tre colpi inferti sono l'impronta della Trinità, il cui numero è ricordato anche dal gesto della mano nella statua di Maderno. Cecilia viene quindi esaltata come vera sposa, poiché ha dato la possibilità a Valeriano di scoprire la vita pura, illuminata dalla fede, sottraendolo al culto pagano, alla tentazione del desiderio e dell'amore carnale: «anch'io le fiamme accolsi, e amando ardei / e di frale desir mi feci oggetto, / ma da te, che nascesti a darmi aita / mi fu tolto l'ardir, resa la vita» (IV, 24). Nelle parole di Valeriano si 
ribadisce l'adesione ad un programma retorico che oppone le fortune terrene a quelle celesti: i detentori del potere in terra sono soggetti a Dio perché il vero impero è quello celeste, e dovranno anch'essi operare secondo la volontà divina senza affidarsi alla dea fortuna.

Di questo saluto a Cecilia ci è rimasta una seconda versione, testimoniata dal ms. Vat. Barb. Lat. 4069, una copia del Cecilia martire che differisce dal testo a stampa nelle ottave 10-38 del canto IV e per la mancanza dell'Allegoria. ${ }^{10} \mathrm{Nel}$ manoscritto, a c. 8or, all'indicazione «Ottave mutate / nel quarto Canto al v. $\mathrm{x}^{\mathrm{o}}$ » seguono, come in appendice, le ottave accolte nella stampa. Dal confronto tra le ottave manoscritte e quelle a stampa si rileva un rimaneggiamento notevole su almeno tre livelli: disposizione, stile e significato. Le ottave 10-19 del manoscritto sono del tutto improntate a un lessico d'amore che viene radicalmente espunto nella versione a stampa; dall'ottava 20, invece, la narrazione ritorna quasi uguale, se non per la disposizione delle ottave nel testo o per dei cambiamenti lessicali mirati a stemperare la sensualità della bellezza involontariamente tentatrice di Cecilia. È interessante soffermarci sulle nove ottave che, più di tutte, si discostano dal modello proposto poi a stampa.

L'ottava 10 del manoscritto, ad esempio, presenta un incipit uguale alla stampa ma se ne discosta nettamente nella seconda parte. Nel manoscritto vaticano i «lumi chiari» di Cecilia sono, con diversa di prospettiva, $\mathrm{i}$ «fonti amari» che accendono un amore non ricambiato. Valeriano, nella versione del Barb. Lat. 4069, una volta ripreso possesso del suo corpo sulla terra, si rivolge a Cecilia non come devoto ma come un marito sofferente, un amante disperato o un Olindo redivivo, focalizzandosi sui suoi occhi anziché, come si legge nella riscrittura, sul significato del martirio e sui segni salvifici lasciati sul corpo di Cecilia («e visto da tre colpi esser partito / quel collo, ch'adeguar de’ marmi pari / puote in candori l'eccellenza pura, / e rende al suo splendor / la neve oscura // dice [...]» IV, 10). Dalla prospettiva di Valeriano-amante, infatti, gli occhi chiusi di Cecilia sono ciechi non a causa della morte, ma perché accecati dalla ritrosia dell'amante:

Ma Valeriano, ch'a lei visse unito

di giogo marital fra lacci cari, par che di moglie pia grato marito 
già già da lumi spande i fonti amari

e dice: «Oh mio bel sole impallidisco

lumi de lumi miei sereni e chiari

come occhi vi miro, e talpe sete

in ver colui ch'illuminato avete?»

(IV, 10, ms. Barb. Lat. 4069).

Il racconto prosegue, nella versione manoscritta, con un Valeriano-amante che ricorda la bellezza della sua sposa, partendo dall'effetto che ha su di lui il suo sguardo: gli occhi sono porti per «cori erranti», non certamente luci di salus cristiana, e Valeriano si discosta dalla retorica del martirio per rompere in un pianto di dolore per la morte dell'amata:

\author{
Apritevi oh di lumi alte facelle \\ occhi ch'apriste a me del ciel la luce, \\ animati tesori, amate stelle, \\ porto, ch'i cori erranti in porto adduce, \\ Cecilia, oh Dio, le tue sembianze belle \\ crudo ferro ha macchiato e non riluce \\ più quel tuo dì che mi spirava aita \\ e morta miro chi mi diè la vita \\ (IV, 11, ms. Barb. Lat. 4069).
}

Nella versione a stampa, il racconto procede, invece, in una direzione completamente differente e dimentica della Cecilia terrena: questo "secondo" Valeriano ripercorre le tappe del martirio della giovane, dal bagno alla decollazione, e ricorda l'ira di Pluto abbattutasi su di lei.

Nell'ottava successiva del manoscritto si continua a sviluppare, invece, e con climax, l'ambiguità di fondo della bellezza e dell'amore di Cecilia. Cecilia è una «sposa diletta» e soprattutto una «compagnia bramata» e «tempra» dell'«ardore» provato dal suo sposo; come la Sofronia di Tasso, archetipo sul quale ritorneremo, è una «donzella d'alto cor» che non si permette di contraccambiare l'amore a causa del suo voto di castità:

Oh di sangue roman diva ben nata, più de la vita mia più del mio core sposa diletta, compagnia bramata, soave tempra del mio forte ardore, 
refrigerio gradito, anima amata

fida al tuo sposo, e cara al tuo Signore, donzella d'alto cor, di fe' costante, del cielo ancella, e de le stelle amante (IV, 12, ms. Barb. Lat. 4069).

Nella versione a stampa ci si concentra, invece, sull'eroismo di Cecilia, attraverso le parole di Plutone che, come un nuovo Perillo, procura infiniti tormenti a chi lo contraddice ( $\mathrm{O}^{\prime}$ ' di folle regnante affetto insano, / che de la fe', che segui, al par sei cieco, / poiché stimi atterar spirto sovrano, / e maggior danno nel suo male hai teco / del suo duolo una stilla è un oceano, / che gran tempeste d'atre pene ha seco, /e chi l'offende, ne gli altri martori / novo Perillo a sé fabrica ardori» IV, 12).

Nel manoscritto, dall'ottava 13 c'è spazio anche per il tema della gelosia: Cecilia, che trae piacere solo dalla visione in Dio, sta distogliendo lo sguardo dal marito terreno, che la prega di instillare dentro di lui, nuovamente, il fuoco della passione:

\begin{abstract}
Ah tu, mia luce, le tue luci omai, a me non volgi e a versar calde stille provochi in me de' gravi lumi i rai e pur spandi al mio seno alte faville lieta solo in Dio godi, e me non fai di tua vista contento: ah le scintilla del tuo foco gradito in me rinova dè di foco maggior vampa si trova (IV, 13, ms. Barb. Lat. 4069).
\end{abstract}

Nella versione a stampa, invece, l'ottava 13 riprende il motivo del fuoco, ma questo è funzionale alla descrizione delle fiamme del martirio: «Nocer il Bagno a lei Cesar credeo / e nel rinfiammar l'onde, Averno fiero / riportar da la vergine trofeo » $(\mathrm{IV}, 13)$ in quanto le «tempeste d'ardori» possono essere solo quelle dell'inferno.

Nell'ottava 15 del manoscritto, Valeriano insiste nel ricordo dell'amore destato da Cecilia e i suoi «primier contenti / ond'in terra mi strinsi a te d'amore» con un'ambiguità erotica che si rinnova alla vista del corpo morto della sposa, colorandosi di una sottile necrofilia. La donna non è colei che ha 
sconfitto l'inferno, ma è l' «amato oggetto, unico bene» dell'amante e la bellezza del suo corpo tormenta Valeriano come aveva fatto in vita, anzi rinnova le pene amorose ancora più profondamente:
Ah tu, mia luce, le tue luci omai, a me non volgi e a versar calde stille provochi in me de' gravi lumi i rai e pur spandi al mio seno alte faville lieta solo in Dio godi e me non fai di tua vista contento: ah le scintille del tuo foco gradito in me rinova sè di foco maggior vampa si trova (IV, 15, ms. Barb. Lat. 4069).

Nel rifacimento a stampa, si continua, invece, la retorica del martirio con attenzione alla funzione purgativa del bagno (IV, 15).

È poi interessante la struttura dell'ottava 16, dove gli inserti parentetici assecondano l'ambiguità delle parole di Valeriano, con un espediente stilistico molto utilizzato da Costa per sottolineare l'ambivalenza dei registri. Benché appartenente alle sfere celesti, «se dir lice», una volta ripreso possesso della propria «forma terrena» per salutare e accogliere Cecilia in cielo, Valeriano sente rinnovarsi il «mal» che Cecilia aveva provocato nel suo cuore:

\footnotetext{
Benchè de' l'alte stelle io spirto sia e preman i miei piè i campi celesti, pur in forma terrena accolto or fia, che 'l tuo mal (se dir lice) il cor molesti. $\mathrm{E}$ in me (come Ciel suole) anima già le pene no, ma ben gli affetti desti che la terra abitar non si può mai, se non vi sian martiri, o s'odan lai (IV, 16, ms. Barb. Lat. 4069).
}

Nelle ottave accolte a stampa, al contrario, la morte per martirio è descritta come ciò che di più grato ci possa essere: consegna alla vera vita nei cieli ed è una prova di coraggio senza eguali. A Cecilia viene riconosciuto il ruolo di soldato di Cristo e il merito di aver sconfitto l'inferno e Plutone nel nome di Dio, emulando il ruolo della Vergine nell'Apocalisse («E s'al tuo bagno fu l'ardore vinto, / anco il foco purgar la terra suole:/ de le fiamme l'aspetto è a noi gradito, 
/ e caro splende co' suoi raggi il Sole; / vien lo spirto vitale a noi partito / da sommi fochi de l'eccelsa mole, / e tra la sfera del sovrano foco, / il mondo inferior gode il suo loco», IV, 16).

Nel manoscritto, paradossalmente, la morte per martirio è letta nell'ottava successiva come rifiuto d'amore: le «luci» sono avare e la prospettiva soteriologica è del tutto inesistente in questo bagno di ricordi terreni:
Mirate, oh faci mie, luci mie care, specchi de' miei pensieri, archi di fede chi vorria (se potesse) in vene amare stemprar le stelle sue, ch'in voi non vede.
Come chiuse vi scorgo a me sì avare negate in terra i rai de l'alta sede? Se più qui resto io manco e morto spiro, se spenti i lumi del mio sol rimiro (IV, 17, ms. Barb. Lat. 4069).

Prima del ravvedimento di Valeriano segue, nel manoscritto, un progressivo primo piano sulle labbra e sui capelli di Cecilia. A differenza della versione a stampa, che in questo punto si concentra, di nuovo, sul «ferro» e sul collo, segnato dai tre colpi della spada e sul sangue, un «trofeo felicissimo» e motivo di festa dell'Empireo, la versione manoscritta descrive labbra che sono state rubate all'amante per essere donate a Dio, mentre i capelli sono delle «catene», dei «nodi» e dei «dilettosi lacci» che continuano ad irretire il cuore dell'amante. Le chiome di Cecilia dovranno contribuire a fare «del novo ciel seconda reggia» e addobbare, come ghirlande, il luogo del martirio: il risultato, tuttavia, sembra propendere più verso la consacrazione di un tempio d'amore più che di martirio. Per comodità, si riportano, le due ottave 18 e 19 in raffronto:

Labbia, che dia pensar dolci sapeste da fonte di rubino a me tesori, voi che pietose al vostro Dio mi deste, porgete al zelo mio cari ristori, e già, ch'a vita il mio morir traeste, fate, che qui non regnino martori, e la terra per noi, per te si veggia fatta di novo ciel seconda reggia.
E ben il ferro con tre colpi segna la vergine latina al ciel diletta, ch'ogni gran forte del terren più degna ha con invitto cor per lui negletta, che Trino, ed Uno su le Stelle regna, ha triplicata luce in un ristretta, ed è per noi contr'ogni rio furore, gran forza, alta giustitia, e sommo amore. 
E voi, non so, se chiome o pur catene che non fuste al mio cor noiosi impacci, ma per sovrarmi l'alma a dure pene, a miei nodi, e dilettosi lacci spandete luce intorno, e qui serene l'aure al tempio addoggiate, anco tra ghiacci la terra suol aver vampe d'ardore e ne le morti sue rinasce amore (IV, 18-19, ms. Barb. Lat. 4069).
Ond'or tra 'l caldo Bagno io qui ti miro, e tra l'offese del tenareo stuolo, e fra 'l tuo sangue preda del martiro, e trofeo felicissimo del duolo che goder non si può ne l'alto giro la reggia tranquillissima del polo, se pe 'l gran Re de la salute pria, la fragil vita in noi morta non sia (IV, 18-19, CosTA 1644).

L'ottava 20, con incipit «ma che morta dich’io tu viva sei», è identica in entrambe le versioni, se non per la punteggiatura e qualche minimo accorgimento stilistico. Come accennato, le ottave che seguono da qui in poi sono quasi identiche a quelle a stampa, se non per la dislocazione nel testo. Gli eventuali accorgimenti stilistici non ne intaccano né il senso, né il registro, questa volta alto e intento a celebrare il candore e la bellezza spirituale di Cecilia: non più una sposa ritrosa, ma «anima chiara» e «virgineo fiore». Solo in alcuni punti, l'incursione dell'erotico ritorna, seppur affievolita dal contesto che non dà spazio per una deroga vera e propria. Si veda, ad esempio l'ottava 29, che coincide con la 34 della versione a stampa: Cecilia non è «cara» ma è ancora «bella» e potenzialmente capace di ispirare un amore dalle fattezze carnali. La disposizione e il corpo del testo del manoscritto ritornano del tutto conformi a quelli della versione a stampa dall'ottava 36 in poi.

Riflettendo sull'archetipo su cui si modella Valeriano, l'Olindo tassiano, andrà ricordato che anche per Tasso l'amore di Olindo e la castità di Sofronia furono oggetto di lunghi ripensamenti, che portarono all'esclusione della vicenda nella lezione della Conquistata. In un contesto di morte, nel mezzo di un rogo che vede i due amanti schiena a schiena, Olindo proromperà nella sua appassionata dichiarazione d'amore sfogando i propri desideri in una violazione verbale del voto di castità della sua amata («Quest’è dunque quel laccio ond'io sperai / teco accoppiarmi in compagnia di vita? / Questo è quel foco ch'io credea ch'i cori / ne dovesse infiammar d'eguali ardori?» Liberata, II, 33, vv. 5-8) e Costa riprende nella versione manoscritta proprio il segmento tassiano «compagnia di vita» in «compagnia bramata» (IV, 12, ms. Barb. Lat. 4069). 
Concludiamo accennando che Costa, al di là di Olindo, aveva comunque già ragionato su un'amplissima casistica di uomini rifiutati dalle loro donne, rileggendo comicamente il rapporto di non corrispondenza tra gli amanti e il potere della castità in alcuni episodi della sua voluminosa raccolta di lettere amorose. Un esempio da considerare per leggere le ottave espunte alla luce del divertissement costiano sono gli scambi di lettere, ad esempio, attribuiti ad una voce maschile che lamenta la ritrosia della donna amata perché votata a Dio, come nella lettera spedita da un Amante a donna di non libero stato (COSTA 1639, 102-5) e in quella indirizzata da un amante a Rinchiusa donna (ivi, 1059). ${ }^{11}$ In questi scambi l'uomo lamenta la pena di dover vivere in castità per la donna che ama, avendo questa scelto di destinare la gioia del suo frutto alla «severa cura di chi vi creò ben creata» (ibidem, 102) e di vivere la propria libertà nella soggezione all'unico amante a cui si può solo ubbidire: Dio. La cosa importante è che Costa, anche trattando di materia sacra, abbia tentato di creare un ulteriore intreccio di voci e rimandi alle sue opere passate, segnalando a chi si appresta al Cecilia martire, e agli studiosi e studiose dell'autrice, la spia di un'ulteriore recita, di una raffinatissima messinscena che porta alla realizzazione di una conversione che mai ha avuto né luogo né ragione se non nel suo divertissement e fine encomiastico. 


\section{Note}

${ }^{1}$ Sulla fortuna letteraria di Giuditta ed Ester si vedano CosENTINo 2007, 63-77 e i saggi raccolti in BORSETTO 2011; su Giuditta si veda in particolare CARPANÈ 2006.

${ }^{2}$ Da martire a santa, a protettrice di cantanti, musici e strumentisti: le prospettive dalle quali viene letto il rapporto tra Cecilia e la musica devono il tutto ad un'erronea interpretazione dell'antifona latina che apre l'ingresso della messa durante la festa della santa («Cantantibus organis [in realtà "Candentibus organis"], Cecilia virgo in corde suo soli Domino decantabat dicens: fiat Domine cor meum et corpus meum inmaculatum ut non confundar»). In base al verbo della lezione originale, candentibus organis, il senso della frase non vede Cecilia pregare nel suo cuore mentre strumenti musicali suonano durante il banchetto delle nozze, ma cantare durante il martirio e, cioè, sotto la stretta rovente degli strumenti di tortura. Dall'erronea interpretazione dell'antifona proviene anche l'associazione alla santa dell'organetto portatile che l'accompagna dal Medioevo in avanti, sino a rendersi iconica con la Cecilia in estasi di Raffaello (1516), e continuare nelle rappresentazioni seicentesche e settecentesche di Santa Cecilia come strumentista e cantante. Cfr. CONNOLLY 1978; STAITI 2002, 22.

${ }^{3}$ Sulla ricostruzione della vicenda si rimanda a LIROSI 2010.

${ }^{4} \mathrm{Su}$ Santa Cecilia si scrivono melodrammi, sacre rappresentazioni, tragedie e poemi: a titolo sommario si veda, ad esempio, l'opera latina di Battista Spagnoli, Althelmi de sancta Coecilia carmen (1514); la tragedia spirituale Santa Cecilia di Giovanni Alberti (1606); la fortunatissima sacra rappresentazione di suor Cherubina Venturelli stampata dal 1612 al 1685; La Ceciliade, stampata a Parigi, di Niccola Soret (1606) con musica di Abramo Blondet; Il tragico imeneo di Valeriano e Cecilia di Gregorio Urbani (1626); la rappresentazione sacra di Agostino Lampugnani Cecilia predicante (1646). A parte si citano i poemi sacri cinque-seicenteschi di Sebastiano Castelletti (La trionfatrice Cecilia vergine e martire romana, 1594) e di Michelangelo Iacobilli (Santa Cecilia, poemetto sacro, 1614) ristampati più volte nel corso dei due secoli e che, se confrontati con il Cecilia martire di Margherita Costa, offrono degli spunti interessanti da cui emerge nettamente la lettura originale dell'autrice. Per gli estremi bibliografici e un confronto tra questi testi rimando a STELLA 2022.

${ }^{5}$ Sull'epica sacra e agiografica rimando specificatamente a FAINI 2015, 47-52.

${ }^{6}$ Rimando a quanto ho sviluppato in STELLA 2022.

${ }^{7}$ La trascrizione dei testi antichi è stata condotta secondo criteri modernizzanti. Si distingue $u$ da $v$; si muta \& ed et in $e$ (o ed); si riduce $j$ in $i$ e del nesso $i j$ in $i i$; si è modernizzato l'uso degli accenti, della punteggiatura e delle maiuscole; non abbiamo conservato l'h etimologica o pseudo-etimologica; si sono conservati i nessi -ti, -tti davanti a vocale; l'oscillazione delle consonanti scempie o doppie e l'alternanza della forma analitica e sintetica delle preposizioni articolate. In numero romano si riporta il canto, mentre in tondo le ottave di riferimento dall'edizione seicentesca CosTA 1644.

${ }^{8} \mathrm{Cfr}$. il commento dei sonetti in PEVERE 2004, 129.

${ }^{9}$ Cfr. PiAnTONi 2013 per il tema della Maddalena nel Seicento.

${ }^{10}$ Cit. la prima volta da CAPUCCI 1984, 234; cfr. anche COSTA-ZALESSOW 2015, 35.

${ }^{11}$ Sull'epistolario di Costa si veda PIANTONI 2017 e PIANTONI 2018. 


\section{Bibliografia}

\section{ALIGHIERI 1966-1967:}

Dante ALIGHIERI, La Commedia, secondo l'antica vulgata, a c. di Giorgio Petrocchi, Miano, Mondadori, 1966-1967, 3 voll.

ARMELLINI 1891:

Mariano Armellini, Le chiese di Roma dal secolo IV al XIX, Roma, Edizioni del Pasquino, 1891, 2 voll., I.

D’AVILA 1998:

Teresa D'AVILA, Libro della vita, in Teresa d'Avila: opere complete, a c. di Luigi Borriello e Giovanna della Croce, Milano, Paoline Editoriale Libri, 1998, pp. 80-470.

BARBERI 1942:

Francesco BARBERI, Paolo Manuzio e la stamperia del popolo romano (1561-1570), Roma, Gela, 1942.

BELLINI 2004:

Eraldo BELLINI, Petrarca e i letterati barberiniani, in Petrarca in barocco. Cantieri petrarchistici, Due seminari romani, a c. di Amedeo Quondam, Roma, Bulzoni, 2004, 2 voll., I, pp. 167-97.

BELLINI 2009:

Eraldo BELLINI, Stili di pensiero nel Seicento italiano. Galileo, i Lincei, i Barberini, Pisa, Edizioni ETS, 2009, pp. 179-88.

\section{BENEDETTI 1997:}

Laura BENEDETTI, La sconfitta di Diana. Un percorso liberata, Ravenna, Longo, 1997.

\section{BORSETTO 2011:}

Giuditta e altre eroine bibliche tra Rinascimento e Barocco, Atti del seminario di studio, Padova, 10-11 dicembre 2007, a c. di Luciana Borsetto, Padova, Padova University Press, 2011.

LO BIANCO 2007:

Anna Lo BiAnco, La Santa Cecilia di Stefano Maderno, in Santa Cecilia in Trastevere, a c. di Carlo La Bella, Roma, Palombi, 2007, pp. 159-72.

CARMINATI 2018:

Clizia CARMINATI, Vita e morte del Cavalier Marino, Bergamo, I libri di Emil, 2018.

CARPANÈ 2006:

Lorenzo CARPANÈ, Da Giuditta a Giuditta: l'epopea dell'eroina sacra nel barocco, Alessandria, ed. dell'Orso, 2006.

CONNOLLY 1978:

Thomas H. Connolly, T. H., The Legend of St Cecilia, I. The Origins of the Cult, in «Studi musicali», 7 (1978), pp. 3-37. 
COSENTINO 2007:

Paolo CosEnTINo, 'Belle, caste e magnanime': le eroine bibliche di Federico Della Valle, in Il mito nel testo. Gli antichi e la Bibbia nella letteratura italiana, a c. di Katia Cappellini e Lorenzo Geri, Roma, Bulzoni, 2007, pp. 63-77.

CosTA 1638:

Margherita CosTA, La chitarra, Francoforte, Daniele Wastch, 1638.

COSTA 1639:

Margherita CosTA, Lettere amorose Lettere amorose della Signora Margherita Costa, Romana, Venezia, s.i.t., 1639.

COSTA 1644:

Margherita Costa, Cecilia Martire. Poema Sacro di Margherita Costa romana all'emin.mo prencipe Francesco card. Barberino, Roma, Mascardi, 1644.

COSTA-ZALESSOW 2015:

Natalia Costa-Zalessow, Voice of a Virtuosa and Courtesan. Selected Poems of Margherita Costa, New York, Bordighera Press, 2015.

FAINI 2015:

Marco FAINI, La poetica dell'epica sacra tra Cinque e Seicento in Italia, in «The Italianist», 35 (2015), pp. 27-60.

FUMAROLI 1995:

Marc FUMAROLI, L'enciclica poetica di Urbano VIII Barberini, in La scuola del silenzio: il senso delle immagini nel XVII secolo, Adelphi, Milano, 1995, pp. 147-49.

GoODSON 2007:

Caroline J. Goodson, Material Memory: Rebuilding the Basilica of St. Cecilia in Trastevere, Rome, in «Early Medieval Rome», 15 (2007), 1, pp. 2052 .

GOETHALS 2017a:

Jessica GoETHALs, The Bizarre Muse: the Literary Persona of Margherita Costa, in «Early modern women», 12 (2017), 1, pp. 48-72.

GoETHALS 2020a:

Jessica GoETHALS, The Singing Saint: The Martyrdom of Saint Cecilia in Seventeenth-Century Literature and Theatre, in "Women language literature in Italy: Donne lingua letteratura in Italia», 2 (2020), pp. 43-61.

HANNING 2004:

Barbara RUSSANO HANNING, From Saint to Muse: Representations of Saint Cecilia in Florence, in «Music in Art», 29 (2004), 1-2, pp. 91-103.

MARINO 1987:

Giovan Battista MARINO, Rime amorose, a c. di Ottavio Besomi e Alessandro Martini, Modena, Panini, 1987.

PEVERE 2004:

Fulvio PEVERE, "Mirti amorosi" ed "eterni lauri": forme del petrarchismo nella poesia di Fulvio Testi, in Petrarca in barocco. Cantieri petrarchistici, Due seminari romani, a c. di Amedeo Quondam, Roma, Bulzoni, 2004, 2 voll., I, pp. 123-50. 
PIANTONI 2013:

Luca PiAnTONI, 'Lasciva e penitente'. Nuovi sondaggi sul tema della Maddalena nella poesia religiosa del Seicento, in «Studi secenteschi», 54 (2013), pp. 25-48.

\section{PIANTONI 2017:}

Luca PIANTONI, L'epistolario amoroso di Margherita Costa, in L'Italianistica oggi: ricerca e didattica, Atti del XIX Congresso dell'ADI - Associazione degli Italianisti (Roma, 9-12 settembre 2015), a c. di Beatrice Alfonzetti, Teresa Cancro, Valeria Di Iasio, Ester Pietrobon, Roma, Adi editore, 2017, pp. 1-5.

PIANTONI 2018:

Luca PIANTONI, Le lettere amorose di Margherita Costa tra sperimentalismo e 'divertissement', in «Studi Secenteschi», 59 (2018), pp. 33-52.

RICCIO 1889:

Luigi RICcIO, Nuovi documenti sull'incendio vesuviano dell'anno 1631 e bibliografia di quella eruzione, in «Archivio storico per le province napoletane», 14 (1889), 3-4, pp. 513-21.

RADEGLIA 2007:

Daila RAdeglia, La Cappella del Bagno, in Santa Cecilia in Trastevere, Santa Cecilia in Trastevere, a c. di Carlo La Bella, Roma, Palombi, 2007, pp. 145-56.

STAITI 2002:

Nico STAITI, Le metamorfosi di Santa Cecilia: l'immagine e la musica, Lucca, Limm, 2002.

STELLA 2022:

Clara Stella, Il Cecilia Martire di Margherita Costa (1644): pentimento e renovatio alla corte dei Barberini, in «Studi secenteschi», 62 (2021), c.s.

TASSO 1951:

Torquato TASSO, Il Mondo creato, a c. di Giorgio Petrocchi, Firenze, Le Monnier, 1951.

TASSO 2009:

Torquato TASso, La Gerusalemme liberata, a c. di Franco Tomasi, Milano, Bur, 2009.

WEAVER 2009:

Elissa B. WEAVER, The "Rappresentazione di Santa Cecilia Vergine e Martire" Written by Suor Cherubina Venturelli, in Scenes from Italian Convent Life: An Anthology of Convent Theatrical Texts and Contexts, a c. di Carolina Bandurski, Ravenna, Longo, 2009, pp. 37-51. 